\title{
Multiple Double XTEM Sample Preparation of Sub-10 nm Diameter Si Nanowires
}

\author{
L. M. Gignac, S. Mittal, S. Bangsaruntip, G. M. Cohen, and J. W. Sleight
}

IBM T. J. Watson Research Center, 1101 Kitchawan Rd., Yorktown Heights, NY 10598

At Microscopy \& Microanalysis 2009, we demonstrated that a transmission electron microscope (TEM) cross-section (X-TEM) sample of a specific, electrically tested sub-30 nm diameter Si nanowire $(\mathrm{NW})$ semiconductor device could be prepared from a previously made X-TEM sample.[1] In this work, two physical measurements: the device gate length (from X-TEM sample 1) and NW diameter (from X-TEM sample 2 sectioned $90^{\circ}$ from X-TEM 1) could be performed from the same electrically tested NW device. Here, the barrier where one or the other of these physical measurements had to be chosen to understand the electrical measurements of a specific device was broken. Now we will show how this initial technique is further extended to allow multiple X-TEM samples to be prepared from an existing X-TEM sample containing specific, electrically tested sub$10 \mathrm{~nm}$ diameter Si NW devices.

In Fig. 1a, a scanning electron microscope (SEM) image is shown of X-TEM sample 1 that contains a series of six electrically tested, sub-10 nm diameter Si NW devices. This X-TEM sample was prepared in a dualbeam focused ion beam (DB-FIB) using in-situ liftout sample preparation with the sample oriented along the NW direction. X-TEM sample 1 was used to measure the gate lengths and profiles for all six Si NW devices, see Fig. 1b for a TEM image of Si NW device 3. X-TEM sample 1 was then placed back in the DB-FIB where four of the six NW's were targeted for re-sectioning. $\mathrm{X}$-TEM sample 1 was detached from the original TEM grid and attached to an in-situ liftout probe. A second in-situ liftout grid was rotated at $90^{\circ}$ from the original grid direction and the targeted regions in X-TEM sample 1 were then reattached to separate fingers on the second grid. This method is similar to FIB atom probe sample preparation. All attach/detach/reattach steps were done with the stage at $52^{\circ}$ so that the ion beam could cleanly cut at $90^{\circ}$ from the X-TEM 1 sample surface and a tight pitch between reattach samples could be obtained. Once on the grid, the targeted regions were thinned to TEM transparency using the FIB ion beam.

Fig. 2 shows a series of TEM images from the four X-TEM 2 samples. The pitch between consecutive X-TEM samples was $1.1 \mu \mathrm{m}$. Successful second X-TEM samples of sub-10 $\mathrm{nm}$ diameter Si NW's were obtained for three of the four regions. Considerable bend in the X-TEM 2 samples is seen which could have been caused from final thinning during XTEM 1 preparation and/or stress generated from encapsulating the samples with FIB deposited e-beam Pt. A $13 \mathrm{~nm}$ thick damage layer was seen in the Si substrate from X-TEM 1 sample preparation. The Si NW diameters ranged from 8.0 to $9.7 \mathrm{~nm}$. Fig. 3 shows high resolution TEM (HR-TEM) images of Si NW 3 taken both parallel and perpendicular to the NW direction demonstrating the ability to obtain HR-TEM images of an identical region at directions that are $90^{\circ}$ from each other. This method proved to be especially useful to study process variation in an array of adjacent devices.

\section{References}

[1] L.M. Gignac, S. Mittal, S. Bangsaruntip, G.M. Cohen, and J.W. Sleight, Microscopy \& Microanalysis, 15, 330 (2009). 


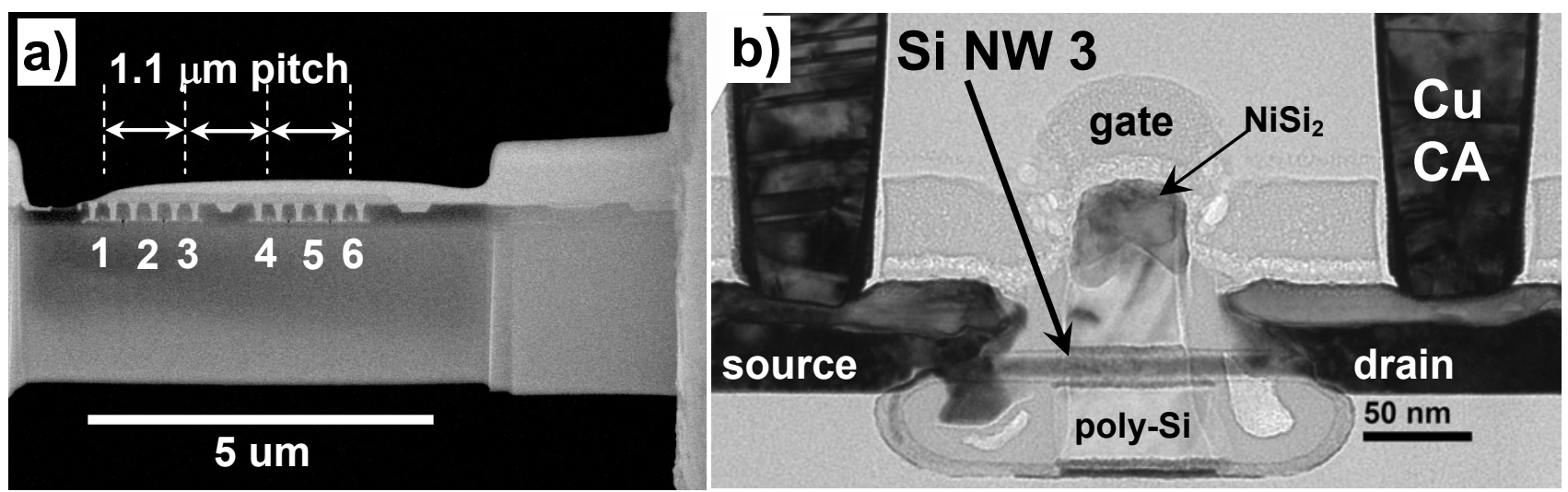

FIG. 1. a) SEM image of X-TEM 1 containing six, electrically tested sub-10 nm diameter Si NW devices with the NW locations labeled 1-6 and b) TEM image from X-TEM sample 1 of NW 3.

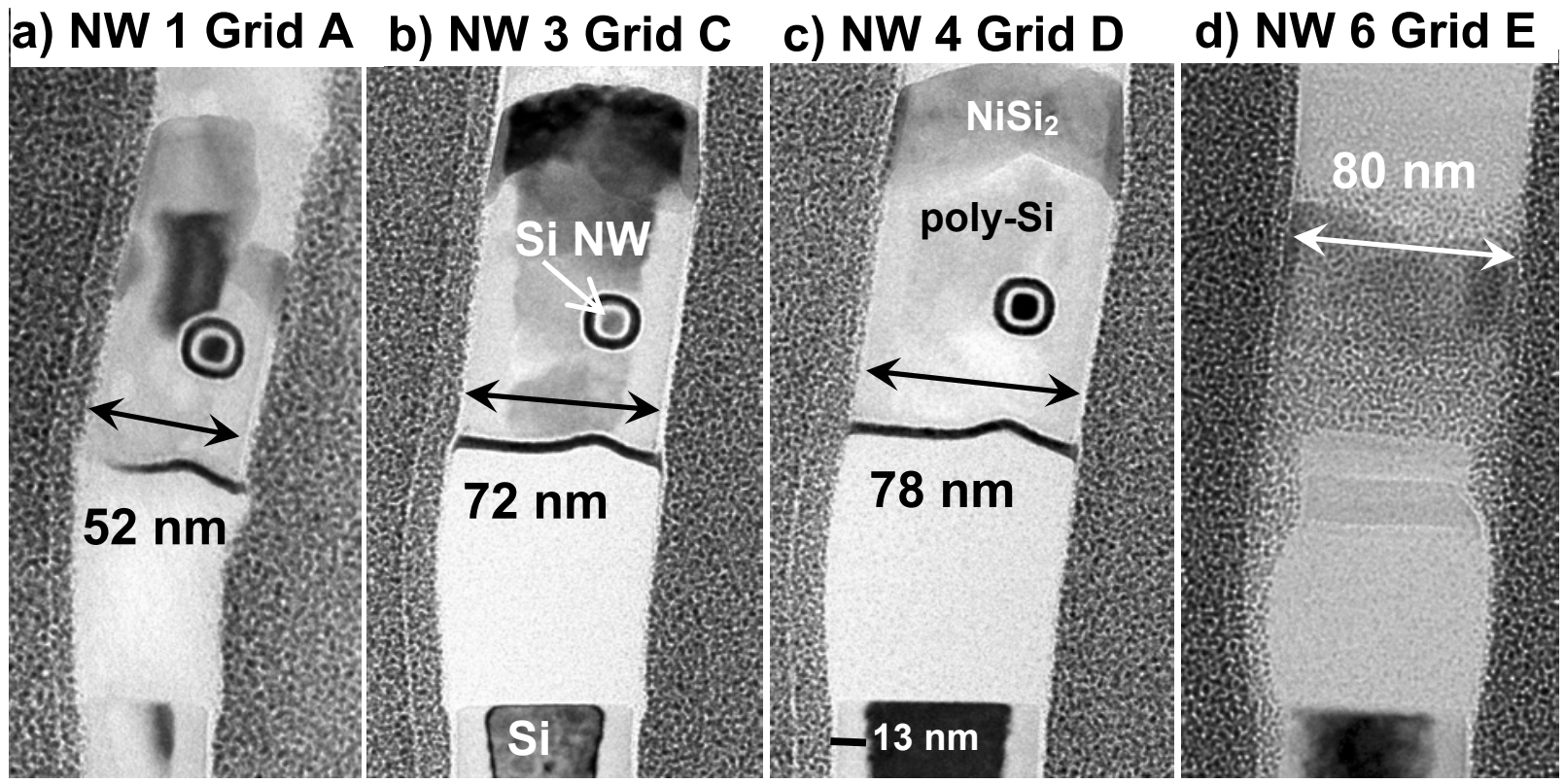

FIG. 2. TEM images from X-TEM 2 samples: a) NW 1, b) NW 3, c) NW 4 and d) NW 6 which was missed while sectioning.

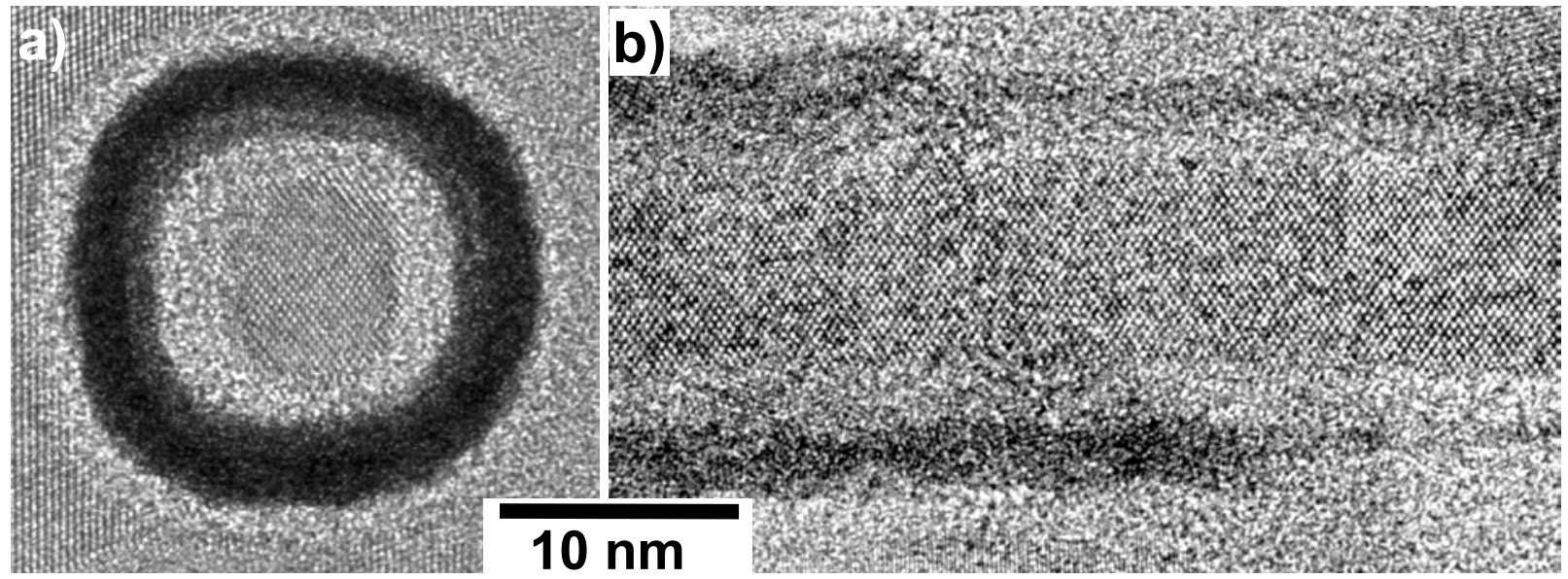

FIG. 3. HR-TEM images of NW 3 sectioned a) perpendicular and b) parallel to the NW. 